\title{
A New Payment Rule for Core-Selecting Package Auctions*
}

\author{
Aytek Erdil ${ }^{\dagger}$ \\ Paul Klemperer ${ }^{\ddagger}$
}

November 2009

\section{A revision of this paper appeared in Journal of the European \\ Economic Association, 2010, vol: 8, iss: 2-3, 537-47.}

the most recent version is at WWW.paulklemperer.org

\begin{abstract}
We propose a new, easy-to-implement, class of payment rules, "Reference Rules," to make core-selecting package auctions more robust. Small, almostriskless, profitable deviations from "truthful bidding" are often easy for bidders to find under currently-used payment rules. Reference Rules perform better than existing rules on our marginal-incentive-to-deviate criterion, and are as robust as existing rules to large deviations. Other considerations, including fairness and comprehensibility, also support the use of Reference Rules.

Keywords: multi-object auction, core, combinatorial auction, package auction, core-selecting auction, Vickrey auction, Vickrey, simultaneous ascending auction, robust design. (JEL: D44, C71)

*We are grateful to Jeremy Bulow, Kalyan Chatterjee, Myeonghwan Cho, Peter Cramton, Bob Day, Gerhard Dijkstra, Jacob Goeree, Rocco Macchiavello, Daniel Marszalec, Meg Meyer, Paul Milgrom, Marco Pagnozzi, David Parkes, an anonymous referee, the editor and many other friends and colleagues for helpful discussions and advice. Erdil thanks the Hausdorff Research Institute in Bonn for its hospitality during the Trimestr Program on Mechanism Design.

$\dagger$ Faculty of Economics, University of Cambridge. E-mail: aytek.erdil@gmail.com

${ }^{\ddagger}$ Department of Economics, Oxford University. E-mail: paul.klemperer@economics.ox.ac.uk
\end{abstract}




\section{Introduction}

Day and Milgrom (2008) recently proposed a novel multi-object auction form -the "core-selecting package auction" - that seems sufficiently attractive, in particular in its handling of complementarities between objects, that it has already been adopted by regulators in several countries. The United States planned to use it for auctioning airport takeoff and landing slots, and the United Kingdom and other European countries have used it for auctioning radio spectrum.1. However Day and Milgrom's original work did not completely specify the auction's payment rules. This paper helps fill that gap.

A core-selecting auction takes sealed bids, identifies the "efficient" allocation (i.e., the allocation that would be value-maximising if all bids were actual values), and chooses associated payments so that the final (non-negative) payoffs are in the core (i.e., no set of bidders can join with the seller to form a "blocking coalition"). That is, a core-selecting auction allocates goods in the same way as a Vickrey-Clarke-Groves (henceforth, Vickrey) auction but substitutes core payments for Vickrey payments.

Economic theorists have, of course, traditionally favoured Vickrey pricing because bidders' dominant strategies are then to bid their actual values for packages in simple private-value environments $:^{2}$ However, Vickrey pricing can lead to very low revenues, extreme incentives to merge and demerge, collusive possibilities that are hard to guard against (in particular, successful collusion requires only two bidders to participate), and the possibility that the auctioneer and/or bidders can gain from using "shills" (see, e.g., Ausubel and Milgrom (2006)). Substituting core pricing for Vickrey pricing mitigates these problems.

Of course, substituting core pricing for Vickrey pricing also creates incentives for bidders to deviate from bidding their values. Day and Milgrom therefore suggested using minimum-revenue core (MRC) pricing to "minimise" bidders' incentives to deviate from "truthful bidding" (when each bidder knows all other bidders' actual values and assumes the others are bidding their true values); specifically, Day and Raghavan

\footnotetext{
${ }^{1}$ The radio spectrum auctions mostly have a clock-proxy first stage based on Ausubel et al. (2006). We do not address issues about this stage in this paper. The planned airport-slot auction was canceled.

${ }^{2}$ This result assumes no budget constraints, no worries about revealing information to third parties, no externalities between bidders (unless bidders can make bids that depend on other bidders' allocations), etc.
} 
(2007) showed that among all core-payment vectors, the MRC selections minimise the sum-across-bidders of each bidder's maximum possible gain from unilaterally deviating from bidding her actual value.

However, unless the Vickrey point is in the core, the MRC is typically not a single point, and in this case Day and Milgrom provide no guidance about how to choose the payment vector. In realistic environments, bidders' payments can be extremely sensitive to this choice $3^{3}$ The planned auctions to date of which we are aware follow Day and Cramton's (2008) suggestion of selecting the MRC point that is closest in Euclidean distance to the Vickrey payments. But the justification for this "Vickreynearest" rule is unclear, since all the points in the MRC minimise the sum of bidders' incentives to deviate from truth-telling. ${ }^{4}$

One can also question the significance of a bidder's maximum possible gain from a deviation. Achieving this would require the bidder to deviate (arbitrarily close) to the point at which her allocation would change, so arbitrarily small changes by other bidders could cause her a net loss, and even a perfectly-informed bidder would be taking a risk.

By contrast, a bidder that reduces her winning bid for a package by a small amount below her actual value obtains an almost-riskless profit in any core-selecting auction in which payments are increasing in winning bids. Thus, for example, for the Vickreynearest rule an $\epsilon$ reduction gives the bidder a first-order gain by reducing her payment for her current allocation by (the order of) $\epsilon$ with probability close to 1 whenever the Vickrey point is outside the core (the payment is unaffected when the Vickrey point is in the core); and generates only a second-order loss, since with probability (of order) $\epsilon$ she would change the allocation, but that could happen only when her actual value is at most $\epsilon$ more than what she needs to pay to be a winner. Furthermore, in a complex environment, bidders are unlikely to understand the full space of alternatives, but may

\footnotetext{
${ }^{3}$ Experiments simulating real contexts have led to outcomes in which total bidders' payments (i.e., total MRC revenues) are of the order of ten times Vickrey payments which means different MRC-selecting payment rules might reallocate as much as $90 \%$ of total payments among bidders. Cramton (2009, p.25) reports experiments in which subjects typically bid truthfully in a Vickreynearest MRC-selecting package auction.

${ }^{4}$ Alternatively, if the Euclidean distance from the Vickrey payments is a better aggregate measure of incentives to deviate from truth-telling, as Parkes et al (2001) suggested, then why restrict attention to the MRC? (The MRC may not include the core point that minimises the Euclidean distance, see Day and Raghavan, 2007. Note 19 give a simple example.)
} 
have a clearer view of where and how to gain from smaller deviations. So marginal incentives for "truth telling" probably matter most for a mechanism's robustness.

This paper, therefore, argues that payment rules for core-selecting auctions should minimise marginal incentives to deviate from "truth-telling."

Specifically, we propose a class of "Reference Rules" in which bidders' payments are, roughly speaking, determined independently of their own bids as far as possible. More precisely, a reference rule selects the MRC payments that are closest to a reference point (a vector of reference payments) chosen by the auction designer, based on any information available to her except the winners' bids (in particular, the reference point can be based upon the losers' bids).

We show that in the environments we consider, there always exists a Reference Rule that dominates the Vickrey-nearest rule in the sense that the Reference Rule has a lower sum-across-bidders of marginal deviation incentives for all possible valuation vectors (and has a strictly lower sum for some valuation vectors) 5 A regulator who has any prior information about the distributions of bidders' valuations can, of course, do even better (in expected terms) by choosing an appropriate Reference Rule. Alternatively, a regulator can pursue an objective such as "fairness," by using a Reference Rule that implements "minimally discriminatory" prices that are as close to equal as possible for identical packages.

Of course, the standard economists' approach to choosing the best payment rule would be to ask which rule is most socially-efficient when bidders play (Bayesian-Nash) equilibrium in response to the rule. Erdil and Klemperer (2009) show the "Vickreynearest" rule is the ex-ante welfare-maximising MRC-selecting rule in probably the simplest example possible, with uniform priors, and when -crucially- the values of the runner-up bids are commonly known in advance. ${ }^{6}$ But this result does not extend

\footnotetext{
${ }^{5}$ Our use of a sum-across-bidders criterion follows most of the literature. Reference Rules also perform well on the probability-that-bidders-have-zero-marginal-incentive criterion. (This criterion might suit a regulator keen to minimise the probability of bidder-regret, and is in the spirit of Lubin and Parkes (2009); Parkes et al.'s (2001) "Small rule" likewise maximises the number of bidders who have zero incentive to deviate.) The Vickrey-nearest rule sometimes performs well on a minimaxmarginal-incentives criterion, but not always (and this criterion in any case seems inconsistent with selecting from the MRC, rather than from the "bidder-optimal core" -see Erdil and Klemperer, 2009).

${ }^{6}$ See also the Appendix.
} 
beyond this unrealistically simple environment,7 so it provides only limited support for the Vickrey-nearest rule, or any other rule. Finding the "optimal" rule in any more realistic environment seems an intractable problem 8 Furthermore, no real-world regulator could use any rule that depends on detailed information about priors about distributions of bidders' values, and relying on Bayesian-Nash equilibrium responses in these extremely complex many-object environments is in any case questionable.9

The logic that proponents of core-selecting auctions espouse is not, therefore, a simple equilibrium one. They note that bidders use rules of thumb and approximations. If regulators, supported by theory, practice, and experiments, can demonstrate that "truthful" bidding is approximately optimal, then bidders who face huge uncertainties and are usually risk-averse may bid at least roughly this way. Thus Cramton, Day, Milgrom and Raghavan's advocacy of MRC selection was based on the objective of minimising (maximum-possible) incentives to deviate from truthful reporting, rather than on any equilibrium analysis. Our approach in this paper is in a similar spirit, but focusing on marginal -rather than maximal-possible- incentives to deviate from "truth-telling." 10

Section 2 describes the environment, and defines Reference Rules. Section 3 formalises the notion of a bidder's marginal incentive to deviate from "truth-telling." Section 4 shows that extremely simple Reference Rules perform well on our marginal incentives criterion in an elementary example. Section 5 shows the intuition for this example extends to more general environments, and Section 6 concludes.

\footnotetext{
${ }^{7}$ It is particularly implausible that the values of the runner-up bids are commonly known ex ante; in this case it is unclear whether an auction would be needed, or at least whether the runner-up bids would ever actually be made.

${ }^{8}$ For a sense of the difficulties, see Ledyard (2007).

${ }^{9}$ The analysis would anyway be incomplete in ignoring incentives to merge, demerge, bid using multiple entities, collude, etc. It is also unclear why we would want to restrict an equilibrium analysis to rules that select from the MRC, or even from the core. See the Appendix for more discussion of the equilibrium approach.

${ }^{10}$ Similarly, the designers of frequently repeated Internet-advertising auctions are interested in mechanisms (such as the generalised second price auction, Edelman, Ostrovksy, and Schwarz, 2007, Varian, 2007) which yield "locally-envy-free" equilibria, so behaviour is stable in the sense that no bidder can gain from making a small change to his previous (equilibrium) behaviour if all other bidders stick to their previous behaviour.
} 


\section{The Environment}

Consider an auction of two indivisible objects, $X$ and $Y$. Each "type- $X$ " bidder makes (only) a single bid for $X$; each "type- $Y$ " bidder makes a single bid for $Y$; and each "type- $Z$ " bidder makes a single bid for the package of both goods. There are arbitrarily many bidders of each type. Their valuations for the object (or package) they bid for are drawn from intervals $[\underline{x}, \bar{x}],[y, \bar{y}]$, and $[\underline{z}, \bar{z}]$, respectively, with their joint distribution being nonzero everywhere ${ }^{11}$

We write $x_{i}, y_{i}$, and $z_{i}$ for the $i$-th highest among the bids made by type- $X,-Y$, and $-Z$ bidders, respectively. A payment rule $P$ specifies winners' payments as a function of all bids, such that only winners pay and no winner pays more than her bid. We write $P_{j}$ for the payment made by a type- $j$ winner, and $V_{j}$ for her Vickrey payment. For simplicity, we restrict ourselves to rules in which the payment vector is differentiable in all the bids ${ }^{12}$ A monotonic payment rule is one in which every bidder's payment is weakly increasing in her bid.

An outcome, i.e., an allocation and an associated vector of payments, is in the core if no subset of bidders can jointly offer an alternative allocation and a vector of payments which makes the seller and all bidders in the subset weakly better off, and makes at least one of them strictly better off. Slightly abusing terminology, we refer to the payment vectors from core outcomes as core vectors. The Minimum Revenue Core (MRC) consists of the core vectors which minimise the sum of payments among all core vectors.

We consider MRC-Selecting Auctions, so:

(1) the objects are assigned to maximise total value, that is, the highest type- $Z$ bidder wins both objects if and only if $z_{1}>x_{1}+y_{1}$; and the highest type- $X$ and type- $Y$ bidders each win the object they bid for otherwise 13

(2) the payments are such that the outcome is in the MRC.

If the Vickrey vector is in the core, it is the unique MRC vector (it minimises all winners' core payments simultaneously). If, however, the Vickrey point is not in the core, there is in general no core vector which minimises everyone's payment simultaneously.

\footnotetext{
${ }^{11}$ Erdil and Klemperer (2009) discuss some extensions to this model.

${ }^{12}$ This ensures the concept of marginal incentive is well-defined. We also allow the regulator to use randomisations over payment rules, in which case we deal with expected payments.

${ }^{13}$ For simplicity, we ignore ties.
} 


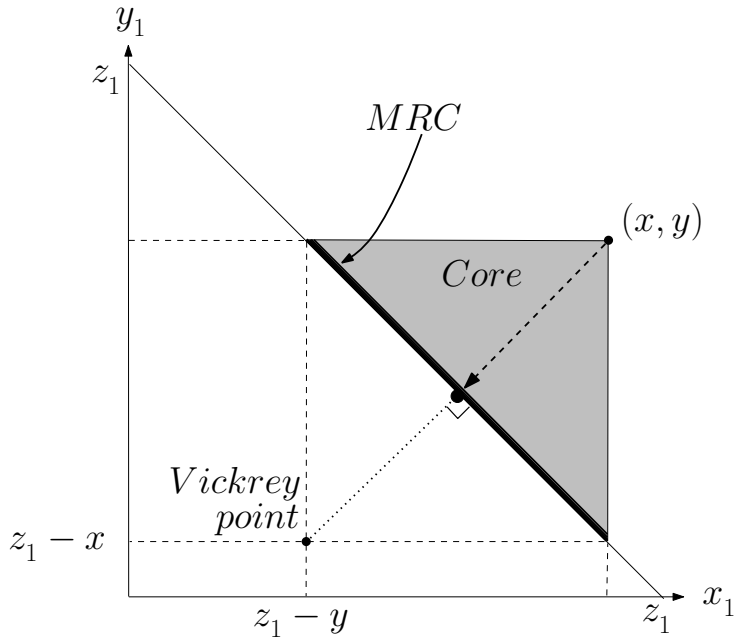

(a) Vickrey point, core, Minimum Revenue Core (MRC), and Vickrey-nearest pricing for a winning bid vector $(x, y)$

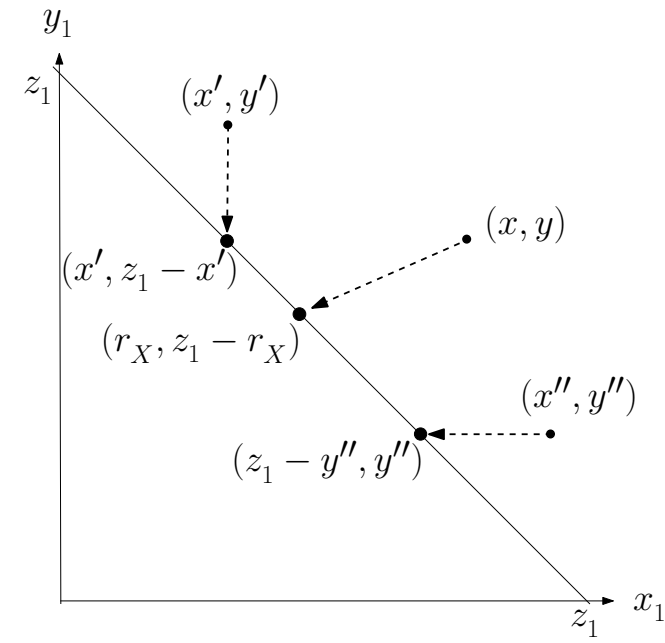

(b) Reference Rule pricing with Reference Payments $\left(r_{X}, z_{1}-r_{X}\right)$ for three different winning bid vectors $(x, y),\left(x^{\prime}, y^{\prime}\right),\left(x^{\prime \prime}, y^{\prime \prime}\right)$

Figure 1: Mapping of winning bid vectors to MRC points for the Vickrey-nearest rule, and the $\left(r_{X}, z_{1}-r_{X}\right)$-reference rule when there is a single bidder of each type. (Note that the Vickrey-nearest payments are sensitive to any perturbations of $(x, y)$, but the reference rule payments are robust to small perturbations of $(x, y)$.)

In an MRC-selecting auction, therefore, if a type- $Z$ bidder wins, she pays $P_{Z}=$ $V_{Z}=\max \left\{z_{2}, x_{1}+y_{1}\right\}$, since the Vickrey vector is then always in the core. If, however, the highest type- $X$ and type- $Y$ bidders are the winners, $V_{X}=\max \left\{x_{2}, z_{1}-y_{1}\right\}$ and $V_{Y}=\max \left\{y_{2}, z_{1}-x_{1}\right\}$, but the sum of their actual payments must be at least $z_{1}$ to be in the core. So if $z_{1} \leq V_{X}+V_{Y}$, they pay $P_{X}=V_{X}$ and $P_{Y}=V_{Y}$. However, if $z_{1}>V_{X}+V_{Y}$, the MRC consists of all payment vectors $\left(P_{X}, P_{Y}\right)$ such that $P_{X}+P_{Y}=$ $z_{1}, x_{2} \leq P_{X} \leq x_{1}$, and $y_{2} \leq P_{Y} \leq y_{1}$ - Figure 1(a) illustrates the Vickrey point, the core, and the MRC, for this case, when there is a single bidder of each type.

The Vickrey-nearest rule picks the point in the MRC that is closest, in Euclidean distance, to the Vickrey vector, so $P_{X}=V_{X}+\left(z_{1}-V_{X}-V_{Y}\right) / 2$ and $P_{Y}=$ $V_{Y}+\left(z_{1}-V_{X}-V_{Y}\right) / 2$. Figure 1 (a) illustrates this rule, when there is a single bidder of each type.

A Reference Rule with Reference Payments $r$ picks the closest point of the MRC to $r$, in which $r$ is independent of the winners' bids, but can depend on any other information (e.g., losers' bids), or criteria (e.g., equal prices for identical objects). 
For example, a possible reference vector is $\left(r_{X}, r_{Y}, r_{Z}\right)$ with $r_{Z}=\max \left\{z_{2}, x_{1}+y_{1}\right\}$; $r_{X}=x_{2}$ and $r_{Y}=y_{2}$ if $z_{1} \leq x_{2}+y_{2}$; but if $z_{1}>x_{2}+y_{2}$ then $r_{Y}=z_{1}-r_{X}$, and $r_{X}$ is any function of $\left\{x_{2}, x_{3}, \ldots, y_{2}, y_{3}, \ldots, z_{1}, z_{2}, \ldots\right\}$ such that $x_{2} \leq r_{X} \leq z_{1}-y_{2}$. With this rule, when the highest type- $X$ and type- $Y$ bidders win, $P_{X}=r_{X}$ and $P_{Y}=r_{Y}$ if both $x_{1} \geq r_{X}$ and $y_{1} \geq r_{Y}$, but $P_{X}=x_{1}$ if $x_{1}<r_{X}$, and $P_{Y}=y_{1}$ if $y_{1}<r_{Y}$. Figure 1(b) illustrates this reference rule when there is a single bidder of each type.

\section{$3 \quad$ Incentives for Small Deviations from Truth-Telling}

We focus on "incentives for small deviations." That is, we assume all bidders bid their actual values, and we then ask what profit increase a winner could have earned if she had made a small change in her bid, holding all other bidders' bids constant.

Let an $\epsilon$-deviation for bidder $j$ with valuation $v_{j}$ be a bid of $v_{j}^{\prime}$ such that $\left|v_{j}-v_{j}^{\prime}\right| \leq$ $\epsilon$. Her marginal incentive to deviate under payment rule $P$ at valuation vector $v$ is then 14

$$
\Delta_{j}^{P}(v)=\lim _{\epsilon \rightarrow 0}\left(\frac{\text { Bidder } j \text { 's maximum profit increase from an } \epsilon \text {-deviation }}{\epsilon}\right) .
$$

Since a type- $Z$ winner always pays her Vickrey payment in a MRC-selecting rule, it is immediate that she has zero incentive to deviate from truthful bidding. We therefore focus on the marginal incentives of type- $X$ and type- $Y$ winners only. For the example of the Vickrey-nearest rule, illustrated in Figure $1(\mathrm{a}), \Delta_{X}=\partial\left(V_{X}+\left(z_{1}-\right.\right.$ $\left.\left.V_{X}-V_{Y}\right) / 2\right) / \partial x_{1}=1 / 2$. And similarly $\Delta_{Y}=1 / 2$.

We say a payment rule $P$ dominates another rule $P^{\prime}$ if the marginal incentive to deviate under $P$ is weakly lower than under $P^{\prime}$ for every vector of valuations, and for each bidder.

It is not hard to see that a monotonic MRC-selecting rule is undominated 15 so a Pareto-like criterion based on marginal incentives does not refine the set of admissible

\footnotetext{
${ }^{14}$ We omit the dependence on $P$ and $v$ when it leads to no confusion. Losing bidders' marginal incentives are, of course, all zero.

${ }^{15}$ If the bid vector is in the MRC, it is the unique MRC point, so all MRC-selecting rules choose it. If $P^{\prime}$ dominates $P$ then along the path on which $x_{1}$ is continuously reduced to $V_{X}$, holding all other bids constant, so that the bid vector is in the MRC, the total decrease in the type- $X$ winner's payment under $P^{\prime}$ cannot be higher than that under $P$. So for any given vector of valuations $P_{X}^{\prime} \leq P_{X}$. Likewise, $P_{Y}^{\prime} \leq P_{Y}$ everywhere. So, since total payments are the same in all MRC-selecting rules, $P$ and $P^{\prime}$ are identical everywhere.
} 


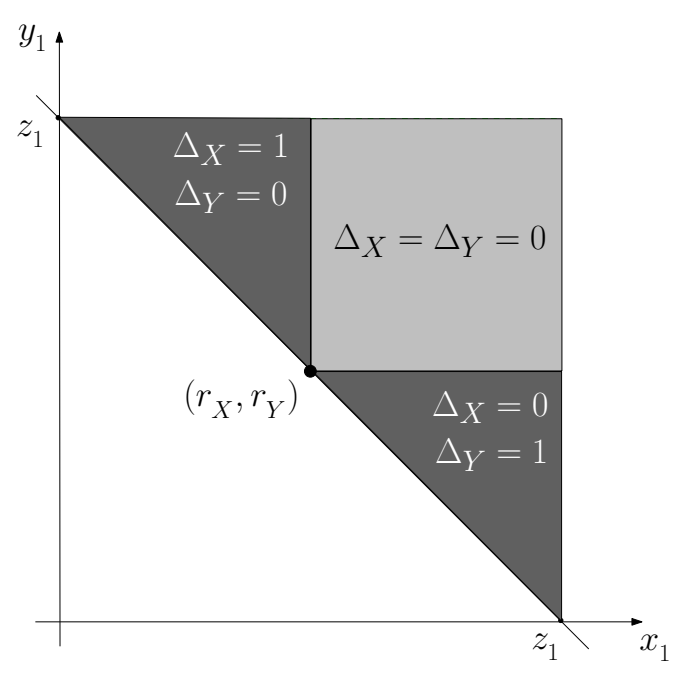

Figure 2: Marginal deviation incentives, $\Delta_{X}$ and $\Delta_{Y}$, of type- $X$ and type- $Y$ winners, for the reference rule with reference payments $\left(r_{X}, r_{Y}\right)$, in the Example.

rules. Previous authors' criterion of minimising the sum-across-bidders of maximum gains from unilateral deviations also restricts the admissible set of rules no further in this environment. But looking at the sum-across-bidders of marginal incentives to deviate does have "bite:"

We say a payment rule $P$ sum-dominates another rule $P^{\prime}$, if the sum-acrossbidders of marginal incentives to deviate is weakly less under $P$ than under $P^{\prime}$ for every vector of valuations.

To illustrate that a reference rule can perform well, and much better than the Vickrey-nearest rule, on this criterion, we now examine a simple special case:

\section{Example}

If there is a single bidder of each type, $z_{1}$ is commonly known, and $x_{1}, y_{1} \in\left[0, z_{1}\right]$, then any reference rule with reference payments $\left(r_{X}, r_{Y}\right)$ such that $r_{X}+r_{Y}=z_{1}$ sumdominates the Vickrey-nearest rule.

Figure 2 shows the marginal deviation incentives, $\Delta_{X}$ and $\Delta_{Y}$, of type- $X$ and type- $Y$ winners, for each valuation vector $\left(x_{1}, y_{1}\right)$, for the reference rule with reference vector $\left(r_{X}, r_{Y}\right)$. In the lightly shaded area, the payments under the reference rule are completely insensitive to small deviations, so the sum of marginal incentives is zero. 
In the darker shaded area, the sum of marginal incentives is one for this reference rule, but for the Vickrey-nearest rule, by contrast, the sum of marginal incentives is one everywhere, as shown in the previous section.

Thus, any reference rule for which $r_{X}+r_{Y}=z_{1}$ improves on the Vickrey-nearest rule in this example. A simple rule of this kind that may also seem "fair" -it selects the MRC payments that are closest to equal- is $r_{X}=r_{Y}=z_{1} / 2{ }^{16}$

The intuition of this example extends to our general environment:

\section{More General Environments}

Proposition. The Vickrey-nearest rule is sum-dominated by a reference rule (with strict domination if $\bar{z} \geq \min \{\bar{x}+\underline{y}, \underline{x}+\bar{y}\}$ ).

Proof. It suffices to specify the reference payments when the winning bids are $x_{1}$ and $y_{1}$. (1) If $z_{1} \geq \bar{x}+y_{2}$ and $z_{1} \geq \bar{y}+x_{2}$, the Vickrey-nearest rule is strictly sum-dominated by any reference rule for which the winners' reference payments are $\left(r_{X}, r_{Y}\right)=\left(q, z_{1}-q\right)$, where $q$ is any function of the losing bids such that $q<\bar{x}$ and $z_{1}-q<\bar{y}$. (2) If $z_{1} \geq \bar{x}+y_{2}$ and $z_{1}<\bar{y}+x_{2}$, the rule with reference vector $\left(x_{2}, z_{1}-x_{2}\right)[17$ strictly sum-dominates the Vickrey-nearest rule. (3) Likewise, when $z_{1} \geq \bar{y}+x_{2}$ and $z_{1}<\bar{x}+y_{2}$, the rule with reference vector $\left(z_{1}-y_{2}, y_{2}\right)$ strictly sum-dominates the Vickrey-nearest rule. (4) Finally, if $z_{1}<\bar{x}+y_{2}$ and $z_{1}<\bar{y}+x_{2}$, then the rule that randomises equally between the reference vectors $\left(x_{2}, z_{1}-x_{2}\right)$ and $\left(z_{1}-y_{2}, y_{2}\right)$, is equivalent, in expectation, to the Vickrey-nearest rule.

The intuition for the result is that conditional on any $x_{2}$ and $y_{2}$, the variables $x_{1}$ and $y_{1}$ are distributed on $\left[x_{2}, \bar{x}\right]$ and $\left[y_{2}, \bar{y}\right]$. Equivalently, changing variables to $x=x_{1}-x_{2}, y=y_{1}-y_{2}, x^{*}=\bar{x}-x_{2}$, and $y^{*}=\bar{y}-y_{2}$, we have that the "winning bids" $x$ and $y$ are distributed on $\left[0, x^{*}\right]$ and $\left[0, y^{*}\right]$-which essentially returns us to the Example of the previous Section 18

\footnotetext{
${ }^{16}$ In this example, Parkes and Ungar's (2000) “iBundle auction” and Ausubel and Milgrom's (2002) equivalent "ascending proxy auction" are equivalent to this rule, but these authors' auctions are not always MRC-selecting.

${ }^{17}$ Note that this rule makes the payment more sensitive to the bidder whose valuation has larger support, so likely higher information rents, consistent with results we would expect from an equilibrium analysis.

${ }^{18}$ Case 1 of the proof corresponds to the trivial extension of the Example to $x^{*}, y^{*} \leq z$, in which $z=z_{1}-x_{2}-y_{2}$ (the Example assumed $x^{*}=y^{*}=z$ ); the reference rule we use corresponds to
} 


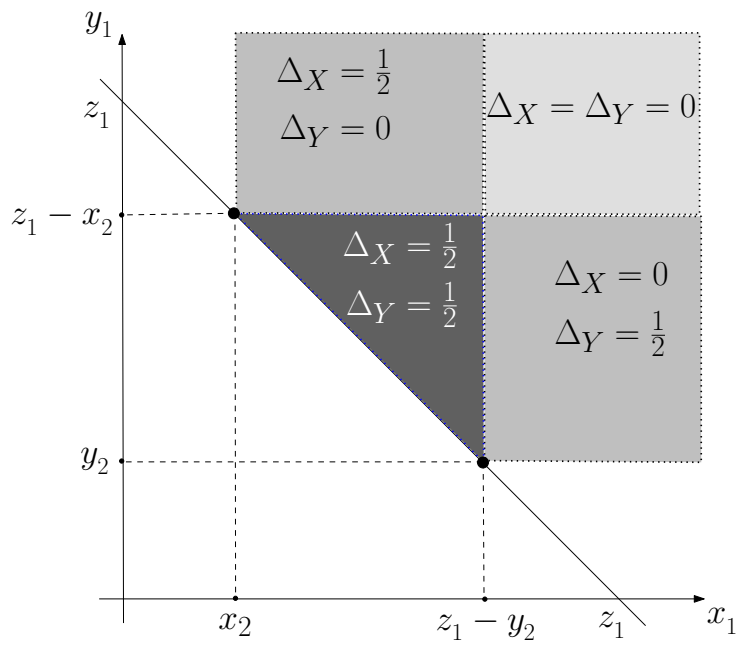

(a) Vickrey-nearest rule

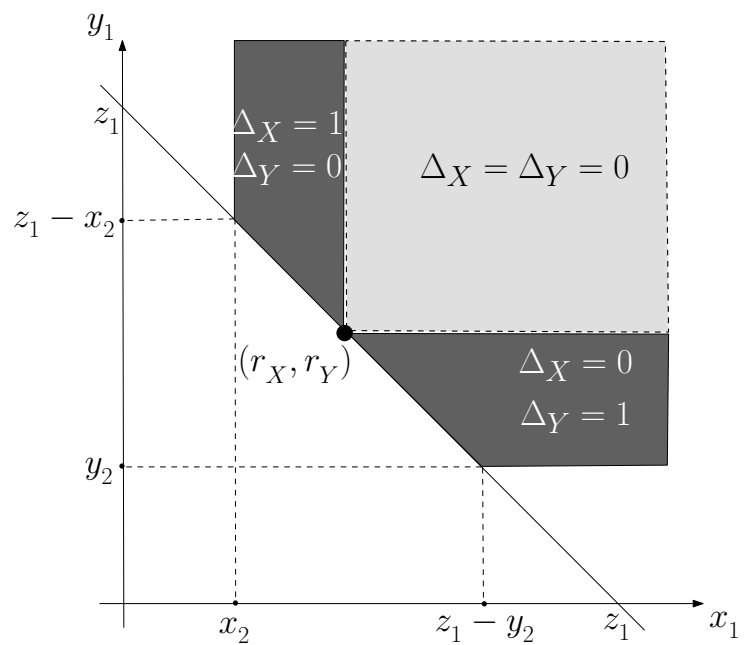

(b) Reference Rule

Figure 3: Marginal deviation incentives, $\Delta_{X}$ and $\Delta_{Y}$, of type- $X$ and type- $Y$ winners, for the Vickrey-nearest rule, and $\left(r_{X}, r_{Y}\right)$-reference rule with $r_{Y}=z_{1}-r_{X}$.

Note the proof shows that the selected reference rule strictly sum-dominates the Vickrey-nearest rule, unless cases (1), (2), and (3) all have zero probability.

Figure 3(a) shows the marginal deviation incentives for each valuation vector $\left(x_{1}, y_{1}\right)$ under the Vickrey-nearest rule. The last part of the proof above uses the fact that these are always identical to the marginal deviation incentives for a reference rule that randomises equally between the reference vectors $\left(x_{2}, z_{1}-x_{2}\right)$ and $\left(z_{1}-y_{2}, y_{2}\right)$. So even a regulator with no information about the distribution of bidders' valuations can pick a reference rule that replicates the Vickrey-nearest rule, and a designer with any distributional information can, of course, do better still by choosing the better-performing of the two reference vectors rather than randomising between them.

Choosing a reference rule with a reference vector that is an appropriately chosen convex combination of the two reference vectors is likely to yield a further improvement: Figure 3(b) shows the marginal deviation incentives under such a reference rule. Although there is now no sum-dominance relationship between the Vickrey-nearest the Example's with reference payments $(q, z-q)$ with $q<x^{*}$ and $z-q<y^{*}$. The remaining cases correspond to the Example with reference vectors $(0, z)$ (case 2, corresponding to $x^{*} \leq z, y^{*}>z$ ); $(z, 0)$ (case 3 , corresponding to $\left.x^{*}>z, y^{*} \leq z\right)$; and equal randomisation between $(0, z)$ and $(z, 0)$ (case 4 , corresponding to $x^{*}>z, y^{*}>z$ ). 
rule in Figure 3(a) and the reference rule in Figure 3(b), the latter clearly does better if there is a high probability of $\left(x_{1}, y_{1}\right)$ lying close to the $45^{\circ}$ line through $\left(r_{X}, r_{Y}\right)$.

These figures thus illustrate how the reference rule can be chosen to achieve robustness over the auction designer's preferred domain, using the information available to her, including what can be learnt from losing bids.

Extending the analysis beyond our two-objects/three-bidder-types environment raises a number of additional issues.

One important issue is that it is not clear whether a core selecting auction should select from the MRC or from the "bidder-optimal core" -the set of core-payment vectors such that no bidder's payoff can be strictly increased without reducing any other's payoff. Although this coincides with the MRC in our environment, it is often a strict superset of it in more general environments 19

Day and Milgrom's original program of selecting among all core-selecting payment rules on the Pareto-like criterion of being undominated in terms of maximal incentives refined the set of admissible payment rules only to the bidder-optimal core-selecting rules. Similarly, if we select among core-selecting rules using Pareto-like criteria based on marginal incentives, we refine to rules selecting from the bidder-optimal core. Minimising the sum-across-bidders of marginal incentives then further refines the set of admissible payment rules to a set including reference rules that choose particular bidder-optimal (but not necessarily MRC) points when possible. ${ }^{20}$

However, the sum-across-bidders of maximum-possible incentives to deviate criterion refines the admissible set from the bidder optimal core to the MRC when these differ. So if minimising both maximum-possible and marginal incentives is desired, we should continue to use our sum-across-bidders of marginal incentives criterion after restricting to MRC points.21

\footnotetext{
${ }^{19}$ For example, if each of three winning bidders has value 2 for any one of three objects $A, B$, and $C$, and a fourth bidder has value 3 for either of the packages $\{A, B\}$, or $\{B, C\}$ (but has no interest in just $B$, or in the package $\{A, C\}$ ), the unique MRC point has payments $1,2,1$ for $A, B, C$, respectively, whereas the bidder-optimal core consists of vectors $(p, 3-p, p)$ with $1 \leq p \leq 2$. The closest core point to the Vickrey payments is $(4 / 3,5 / 3,4 / 3)$.

${ }^{20}$ See Erdil and Klemperer (2009). Likewise, Parkes and Ungar's (2000) and Ausubel and Milgrom's (2002) core-selecting auction is bidder-optimal core- (but not necessarily MRC-) selecting.

${ }^{21}$ By contrast, as discussed in the Introduction, using a Vickrey-nearest rule after restricting to the MRC is harder to justify. Furthermore, with more than two objects, Vickrey-nearest pricing can recreate some of the strange incentives for merger, demerger, and collusion, which core pricing was intended to mitigate. In a simple three-unit example, bidders $i, j$ and $k$ each have value 6 for a single
} 


\section{Conclusion}

Our approach of looking for a rule in which each winner's payment is as far as possible independent of her bid seems in the original spirit of Vickrey pricing. Bidders' marginal incentives for unilateral deviations may be more relevant to whether they bid close to "truthfully" than the maximum-possible incentives to deviate that previous authors focused on; and analysing bidders' marginal incentives suggests Reference Rules are natural payment rules.

Other practical considerations may argue the same way. If the packages being priced comprise identical, or close-to-identical, objects, a Reference Rule can set "minimally discriminatory" prices by specifying reference payments proportional to the number of slots acquired in an airport-slot auction, or proportional to the bandwidth acquired in a radio spectrum auction. ${ }^{22}$ This approach of assigning prices that are as close to equal as possible for equal packages may both seem fair, and also be easy to explain to observers. But our analysis above is clearly only preliminary; much more research remains to be done 23

unit (but no interest in more than one). A "package" bidder has value 12 for the package of all three units (but no use for fewer than three). With truthful bidding, and Vickrey-nearest pricing, $i, j$ and $k$ each pay 4 if they all bid independently, but $i$ and $j$ together pay 9 if they bid as a single entity (and independently of $k$ ) -so merger hurts (and demerger pays) here.

${ }^{22} \mathrm{Or}$ if the core-selecting auction is part of a dynamic package auction, the reference payments might be set proportional to the "activity points."

${ }^{23}$ Nor does our research yet support the more general use of core-selecting auctions. No core-pricing rule fully eliminates the problems that plague the Vickrey auction. For example if two bidders $i$ and $j$ have values for objects $A, B$, and the package $\{A, B\}$, of $2,1,3$, and $1,2,3$, respectively, then allocating $A$ to $i$, and $B$ to $j$ is efficient. The Vickrey payment vector is (1,1). If $i$ increases her bid for $A$ to 3 , the same allocation is chosen with Vickrey payments $(1,0)$. If $j$ also increases her bid for $B$ to 3 , the allocation remains unchanged with Vickrey payments $(0,0)$. Even though all the Vickrey payment vectors are in the core, revenues are non-monotonic in bids (which opens the way for shilling), and collusively raising bids can lower revenues.

While complementarities create problems for the simultaneous ascending auction that is now commonly used for allocating, e.g., radio spectrum (see, e.g., Klemperer, 2004), concerns about this auction's costliness and susceptibility to market power are addressed for "substitutes" environments by Klemperer's $(2008,2010)$ and Milgrom's (2009) recently-proposed auction designs. 


\section{A Appendix}

A Bayesian-Nash equilibrium analysis of Section 4's Example for the special case in which it is common knowledge that $x_{1}$ and $y_{1}$ are independently, identically and uniformly distributed yields:

If bidders choose the bids corresponding to the welfare-maximising Nash equilibrium given the pricing rule, then the welfare-maximising $M R C$-selecting mechanism is Vickrey-nearest pricing.

To understand this result, note that choosing MRC payments for type- $X$ and type$Y$ winners is equivalent to the "public goods" problem of deciding how they should share the cost of raising $z_{1}-\left(V_{X}+V_{Y}\right)$, the amount they are required to pay on top of their Vickrey payments. Since a type- $Z$ bidder pays her Vickrey payment, so has a dominant strategy of bidding her commonly-known valuation, $z_{1}$, it follows that any MRC payment rule $P$ is equivalent (in payoffs for the type- $X$ and type- $Y$ bidders) to a direct-revelation trading rule, where the type- $X$ bidder corresponds to a seller with value $s=z_{1}-x_{1}$, the type- $Y$ bidder corresponds to a buyer with value $b=y_{1}$, and when trade occurs it does so at price $p(b, s)=P_{Y}\left(z_{1}-s, b\right)$. Myerson and Satterthwaite (1983) show that the linear increasing equilibrium of the $1 / 2$-double auction identified by Chatterjee and Samuelson (1983) maximises the expected gains from trade subject to the incentive compatibility and interim individual rationality constraints. So the optimal MRC payment rule would pick $p(b, s)=(b+s) / 2=\left(y_{1}+z_{1}-x_{1}\right) / 2$, which is identical to the type- $Y$ bidder's payment under Vickrey-nearest pricing.

Note however that (i) the Vickrey-nearest rule supports a continuum of other Nash equilibria in this example, and many of these yield very much lower welfare ${ }^{24}$ At least, if we depart from this very special case, there is no obvious way of choosing among equilibria. ${ }^{25}$ (ii) Even the welfare-maximising equilibrium yields not much more of the available surplus than is achieved by the simplest "reference rule." 26 (iii) Even

\footnotetext{
${ }^{24}$ The multiplicity corresponds to the multiplicity of equilibria of the Chatterjee-Samuelson mechanism. The fact that many equilibria yield much lower revenue (as well as lower welfare) than the unique equilibrium of the Vickrey auction casts doubt on the merits of this core-selecting auction.

${ }^{25}$ In our special case it seems reasonable to focus on the unique linear increasing equilibrium.

${ }^{26}$ The latter uses reference payments $r_{X}=r_{Y}=z_{1} / 2, r_{Z}=x_{1}+y_{1}$, and in its simplest equilibrium both bidders bid $z_{1} / 2$ if $v \geq z_{1} / 2$, and zero otherwise, capturing $75 \%$ of the total available surplus, compared with the $84 \%$ captured by the best equilibrium under the Vickrey-nearest rule. See Erdil and Klemperer (2009) for further discussion.
} 
small generalisations seem to render the example intractable ${ }^{27}$

\section{References}

[1] Ausubel, Lawrence, Peter Cramton, and Paul Milgrom (2006). "The clock-proxy auction: a practical combinatorial auction design." In Combinatorial Auctions, edited by Peter Cramton, Yoav Shoham, and Richard Steinberg, MIT Press.

[2] Ausubel, Lawrence, and Paul Milgrom (2002). "Ascending Auctions with Package Bidding." Frontiers of Theoretical Economics, 1:1, Article 1.

[3] Ausubel, Lawrence, and Paul Milgrom (2006). "The lovely but lonely Vickrey auction." In Combinatorial Auctions, edited by Peter Cramton, Yoav Shoham, and Richard Steinberg, MIT Press.

[4] Chatterjee, Kalyan, and William Samuelson (1983). "Bargaining under Incomplete Information." Operations Research, Vol. 31, No. 5, 835-851.

[5] Cramton, Peter (2009). "Spectrum Auction Design." U Maryland Working Paper.

[6] Day, Robert W., and Peter Cramton (2008). "The Quadratic Core-Selecting Payment Rule for Combinatorial Auctions." U Maryland Working Paper.

[7] Day, Robert W., and Paul Milgrom (2008). "Core-selecting package auctions." International Journal of Game Theory, 36:3-4, 393-407.

[8] Day, Robert W., and S. Raghavan (2007). "Fair Payments for Efficient Allocations in Public Sector Combinatorial Auctions." Management Science, 53:9, 1389-1406.

[9] Edelman, Benjamin, Michael Ostrovsky, and Michael Schwarz (2007). "Internet Advertising and the Generalized Second-Price Auction: Selling Billions of Dollars Worth of Keywords." American Economic Review, 97:1, 242-59.

\footnotetext{
${ }^{27}$ In particular, any relaxation of the assumption of common knowledge of $z_{1}$ seems too hard. For a sense of the difficulties, see Ledyard's (2007) impressive analysis of our environment, though without imposing core (or MRC)-pricing constraints.
} 
[10] Erdil, Aytek, and Paul Klemperer (2009). "Alternative Payment Rules in CoreSelecting Package Auctions." Unpublished notes, Oxford University.

[11] Klemperer, Paul (2004). Auctions: Theory and Practice. (The Toulouse Lectures in Economics), Princeton University Press.

[12] Klemperer, Paul (2008). "A New Auction for Substitutes: Central-Bank Liquidity Auctions, "Toxic Asset" Auctions, and Variable Product-Mix Auctions." mimeo, Oxford University.

[13] Klemperer, Paul (2010). "The Product-Mix Auction: A New Auction Design for Differentiated Goods." Journal of the European Economic Association, 8:2-3, $526-536$.

[14] Ledyard, John O. (2007) "Optimal combinatoric auctions with single-minded bidders." EC'0\%: Proc. 8th ACM conference on Electronic commerce, pp. 237-42.

[15] Lubin, Benjamin, and David C. Parkes (2009). "Quantifying the Strategyproofness of Mechanisms via Metrics on Payoff Distributions." Proc. 17th National Conference on Artificial Intelligence (AAAI-00), pp. 74-81.

[16] Milgrom, Paul (2009). "Assignment Messages and Exchanges." American Economic Journal: Microeconomics, 1:2, 95-113.

[17] Myerson, Roger B., and Mark A. Satterthwaite (1983). "Efficient mechanisms for bilateral trading." Journal of Economic Theory, 29, 265-281.

[18] Parkes, David C., Jayant Kalagnanam, and Marta Eso (2001). "Achieving Budget-Balance with Vickrey-Based Payment Schemes in Exchanges." Proc. 17th Int'l Joint Conf. Artificial Intelligence (IJCAI 01), pp. 1161-1168.

[19] Parkes, David C., and Lyle H. Ungar (2000). "Iterative Combinatorial Auctions: Theory and Practice." Proc. 17th National Conference on Artificial Intelligence (AAAI-00), pp. $74-81$.

[20] Varian, Hal (2007). "Position auctions: Theory and Practice." International Journal of Industrial Organization, 25, 1163-78. 